\title{
Automation of fault diagnosis of bearing by application of fuzzy inference system (FIS)
}

\author{
Fateh Harrouche ${ }^{\mathrm{a}}$ And Ahmed Felkaoui \\ Laboratory of Applied Precision Mechanics (LMPA), Institute of Optics and Precision Mechanics, \\ Setif-1 University, 19000 Setif, Algeria
}

Received 7 January 2014, Accepted 15 May 2014

\begin{abstract}
This work deals with the application of the fuzzy logic to automate diagnosis of bearing defects in rotating machines based on vibration signals. The classification tool used is a fuzzy inference system (FIS) of Mamdani type. The vector form of input contains parameters extracted from the signals collected from the test bench studied. The output vector contains the classes for the different operating modes of the experimental study. The results show that; pretreatment data (filtering, decimation,....), the choice of parameters of fuzzy inference system (input variables and output, types and parameters of membership functions associated with different input and output variables of the system, the generation of fuzzy inference rules,...) are of major importance for the performance of fuzzy inference system used as a tool for fault diagnosis of rotating machinery.
\end{abstract}

Key words: Rotating machines / fuzzy logic / fuzzy inference / Fault diagnosis / signal processing

\section{Introduction}

Despite the progress and technological advances in the distributed control and automation of complex processes, monitoring processes remains a very important task which is still largely a manual activity, carried out by operators, especially when it comes to respond to abnormal events. This activity is based on the nature and criticality of defects or misfunctions, affecting economy, environment and security more or less significantly, not only in equipment but also for operators. Especially the fact that manages a large amount of information and needs to act quickly can lead operators to make incorrect decisions, further degrading the situation. Thus, the monitoring systems that integrate tools for monitoring, detection and diagnosis are needed to provide the operator with sufficient criteria for decision-making.

In this context, many approaches are developed for fault detection and diagnosis, by the different scientific communities. The methods differ in the type of a priori knowledge about the process they require. Thus they may be classified, in general, as model-based methods, and methods based on historical data. Methods based on models consider a structural model of the behavior of the process based on fundamental physical principles. These models can be quantitative, expressed as mathematical

\footnotetext{
${ }^{a}$ Corresponding author: harrouchefateh@yahoo.fr
}

equations or qualitative, expressed for example in the form of logical relationships. Methods based on historical data seek to extract information, such as examples or trends within steps from the sensors and actuators, which can identify the behavior of the process. These methods include, among others, statistical methods and classification (or pattern recognition). Among the methods based on historical data, there are methods of pattern recognition based on fuzzy logic. The contribution of fuzzy logic is the possibility of a simulation of the expertise of an operator or designer in the monitoring and process control. From the mid-90s [1-4], applications of fuzzy logic in maintenance of rotating machines begin to appear progressively with the use of different fuzzy classification methods. It was found that several applications in recent years use inference systems based on fuzzy rules, as a tool for classification of faults for diagnosis [5-9].

In our work and in relation to work done in this area, we opt for the application of fuzzy logic for the detection and diagnosis of mechanical faults in electric motors (bearings faults). We use a clustering method with fuzzy inference system (FIS). The results show that; the preprocessing (filtering, decimation,...), and the choice of parameters of fuzzy inference system (input variables and output types and parameters of membership functions associated with different variables input and output of the system, the generation of fuzzy inference rules,...) have a major importance on the performance of fuzzy inference 


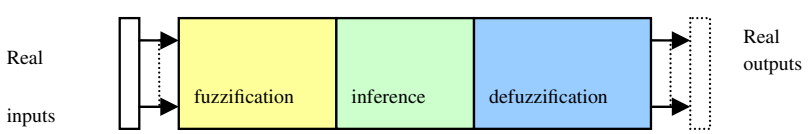

Fig. 1. Stages of the implementation of a fuzzy inference system (FIS).

system used as a diagnosis tool for mechanical faults of electric motors.

\section{Implementation of fuzzy inference system (FIS)}

There are three functional steps in the implementation of a fuzzy inference system (FIS) for the diagnosis, as shown in the diagram (see Fig. 1).

\subsection{Step of fuzzification}

The fuzzification step is to define fuzzy sets for input variables and output for each of these variables must be known a priori: definition of the interval, number of fuzzy sets and shapes of the functions of belonging.

\subsection{Inference step}

This is the stage where we establish the fuzzy rules which allow to reach the exit by the values of the input variables. Each rule consists of premises linked by AND, OR and gives rise to an implication by the operator THEN.

After editing the rules, it remains to calculate the membership degrees of the output variable to all fuzzy sets associated with it.

The aggregation of these rules is an operation that should lead to a single value of the variable output after defuzzification.

\subsection{Defuzzification stage}

This step is to transform the linguistic variable outcome of fuzzy inference system to a digital value. For this, there are three main methods:

- The method of maximum corresponding to the minimum horizontal axis of the ordinate up to the surface. It is little used.

- The method of weighted average.

- The method of centroids is the best. It is simply to calculate the centers of gravity of surfaces.

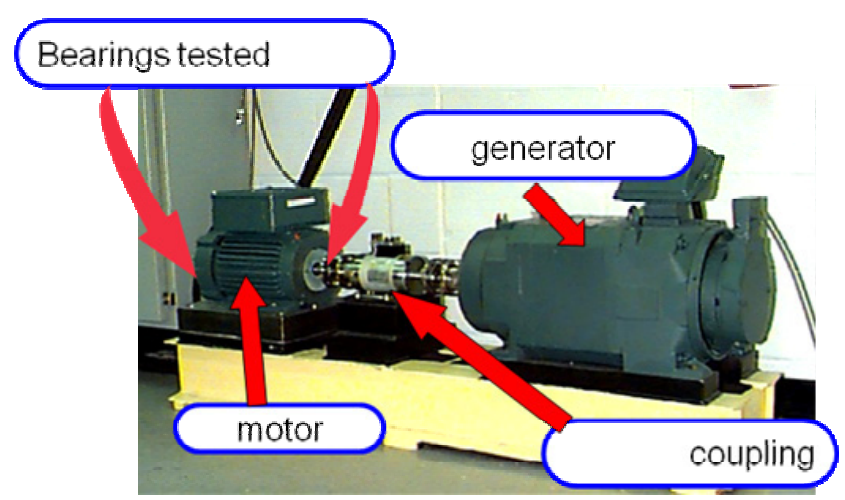

Fig. 2. Bearing test rig.

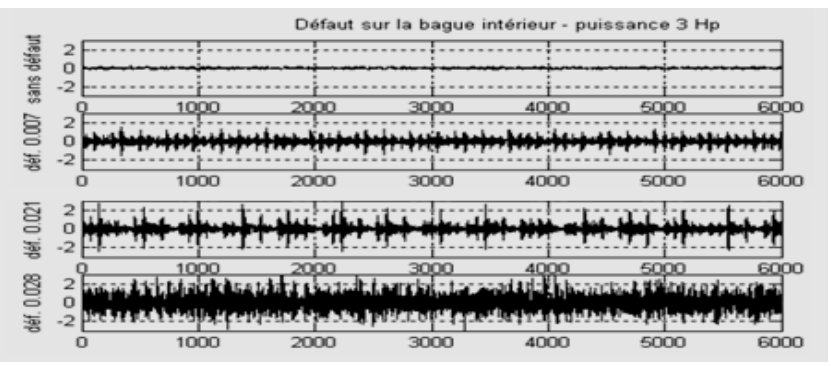

Fig. 3. Representation of time signals for different diameters of the defect on the inner ring.

\section{Test bench}

The website "www.eecs.cwru.edu/laboratory/ bearing/welcome_overview.htm" [10]; provides access to the testing database of ball bearings or bearings normal or defective. As shown in Figure 2 below, the test bench consists mainly of an engine "2HP" (left), a coupling transducer/encoder (center), a generator (right) and control circuits (not shown).

The bearings tested support the motor shaft at both ends (drive side and side fan). Simple defects in the form of points have been created on the bearing test, using electro-discharge machining.

The signals were recorded using accelerometers, which were attached to the cage of the motor magnet. These sensors were placed at the 12 o'clock position "vertical", both sides and fan coupling of the motor.

The signals were recorded examining defects of the inner side coupling under the following conditions:

- The shaft rotates at a speed of $1730 \mathrm{rpm}$.

- The power of the load torque is $3 \mathrm{HP}$.

- Three defects of diameters 0007, 0021, and 0.028 inch were created on the inner race.

- The four signals (states) are drawn on the side studied mating signal without defects and with other defects (defects in three sizes 0007, 0021, and 0.028 inch).

Figure 3, represents the time signals collected on the side of the coupling, for different diameters of defects produced on the inner ring to a torque of $3 \mathrm{HP}$. (HP: Horse Power) 


\section{Constitution of the database}

The database contains four available signals, a signal without defects and three with defects. As the fuzzy inference systems (FIS) require a large database for the classification, and to have enough data, we divided each signal into Kt slices with $N_{M}$ points provided that the number of samples for each slice must be large enough to cover a sufficient number of the largest cycle corresponding to the greatest period (the lowest speed). We have the signals of length 120800 points. The shaft rotates at a speed of $1730 \mathrm{rpm}$.

$$
f r_{\text {shaft }}=\frac{1730}{60}=28.83 \mathrm{~Hz}
$$

It has four characteristic frequencies corresponding to the various elements of the bearing (cage, inner and outer race and rolling elements). The greatest period corresponds to the smallest frequency. In our case, the greatest period is the period of the cage:

$$
f_{\text {cage }}=0.9382 \times 28.83=11.48 \mathrm{~Hz}
$$

- The period:

$$
T_{\text {cage }}=\frac{1}{f_{\text {cage }}}
$$

- The sampling frequency:

$$
f_{s}=12 \mathrm{KHz}=12000 \mathrm{~Hz}
$$

- The sampling period:

$$
\Delta t=\frac{1}{f_{s}}
$$

- The number of points per period:

$$
N_{p}=\frac{T_{\text {cage }}}{\Delta t}=\frac{f_{s}}{f_{\text {cage }}}=\frac{12000}{11.48}=1045.29 \mathrm{pts} \cong 1046 \mathrm{pts}
$$

If we take a factor of 5 , the length will be required for each portion:

$$
N_{p o}=N_{p} \times 5=5230 \mathrm{pts}
$$

If we take a value greater than $2^{n}$

$$
N_{M}=2^{13}=8192
$$

While the number of slices in the case of a recovery of $50 \%, s_{1}=\frac{N_{M}}{2}$ then:

$$
K_{t}=\frac{N_{s}-s_{1}}{N_{M}-s_{1}}=\frac{120800-4096}{4096} \cong 28
$$

So, we got 112 signals, 28 signals for each class.

\subsection{Pretreatment and calculation of indicators}

A signal preprocessing is required to remove all kinds of useless information, and facilitate the task of extracting indicators for monitoring the most relevant database. We chose initially to calculate the following indicators: the STDEV (ET), the crest factors (Fc), the crest-crest (Acc), the factor dissymmetry (skewness $(\mathrm{Ks})$ ) and the flattening factor (Kurtosis $(\mathrm{Ku})$ ) [3,4], and the energy from the spectrum envelope. These indicators can be good indicators for monitoring in the case of the bearings. After a preliminary analysis, we chose to calculate these indicators as follows.

\subsubsection{Temporal indicators}

The time indicators mentioned above are calculated in five frequency bands from the band analysis " $6 \mathrm{KHz}$ ", 4 in adjacent bands of width $1500 \mathrm{~Hz}$, plus the total band. The indicators are the averages of the indicators calculated in the five bands. The bands are then calculated: [0$1500 \mathrm{~Hz}],[1500-3000 \mathrm{~Hz}],[3000-4500 \mathrm{~Hz}],[4500-6000 \mathrm{~Hz}]$ and $[0-6000 \mathrm{~Hz}]$. The signal from each portion has been centered and filtered by filters like band pass.

\subsubsection{Frequency indicators}

The indicators (energy from the spectrum envelope) are calculated in the five (5) frequency band and total frequency bands of width of $1000 \mathrm{~Hz}$, from the spectrum envelope of the different tranches. Frequency indicators are calculated; EBT in the band [0-6000 Hz], EB1 in [0$1000 \mathrm{~Hz}$, EB2 in [1000-2000 Hz], EB3 in [2000-3000 Hz] EB4 in [3000-4000 Hz] and EB5 in [4000-5000 Hz].

- Notes:

- For the division of signals into slices, and the calculation of time and frequency indicators, a program in Matlab is designed for this purpose.

- Figures 4-14 graphically represent the variation of the 11 indicators based on 112 observations (number of slices). Note that the numerical values of the three indicators ET, EB2 and EB5 corresponding to Figures 4,11 , and 14 are divided into 4 levels completely separated from each other, these levels correspond to the four operating conditions studied, and therefore the three indicators ET, EB2 and EB5 are most relevant for classification.

\subsection{Constitution of vector form}

The vector form consists of a number of indicators selected from the previously calculated indicators. The components of the vector form representing the input variables of the fuzzy inference system (FIS) are chosen 


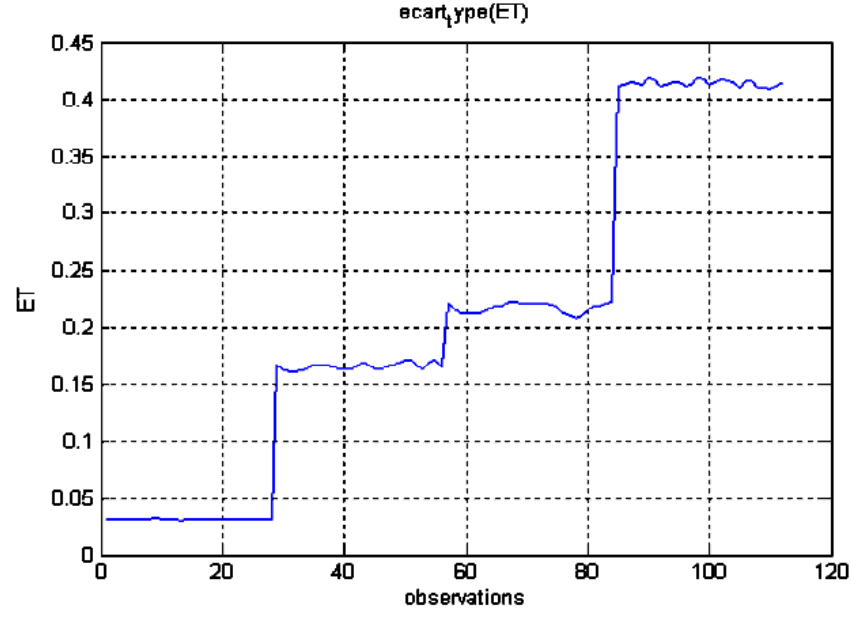

Fig. 4. Variation indicator (ET).

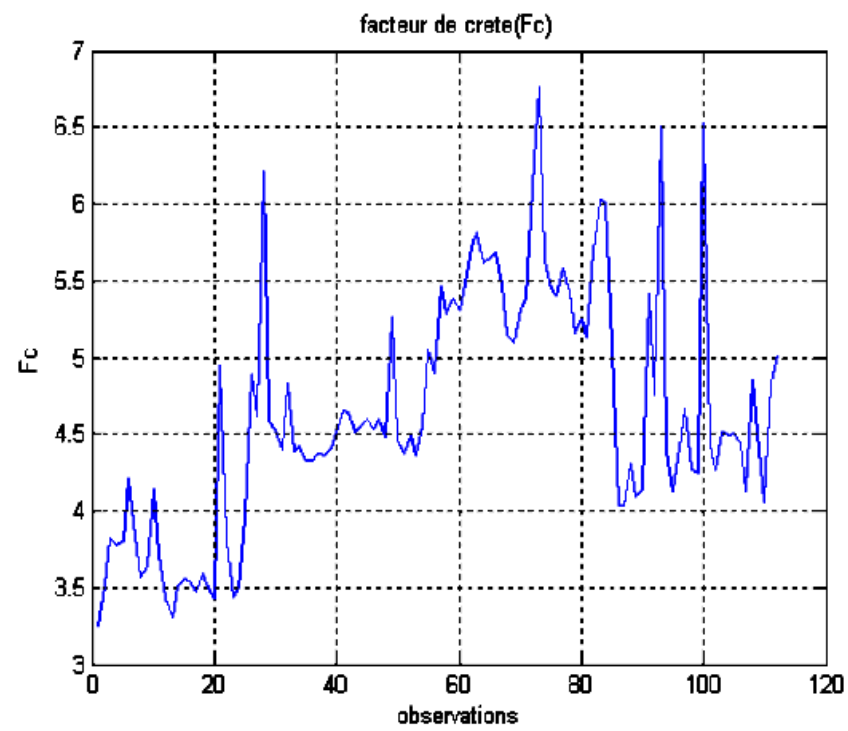

Fig. 5. Variation indicator (FC).

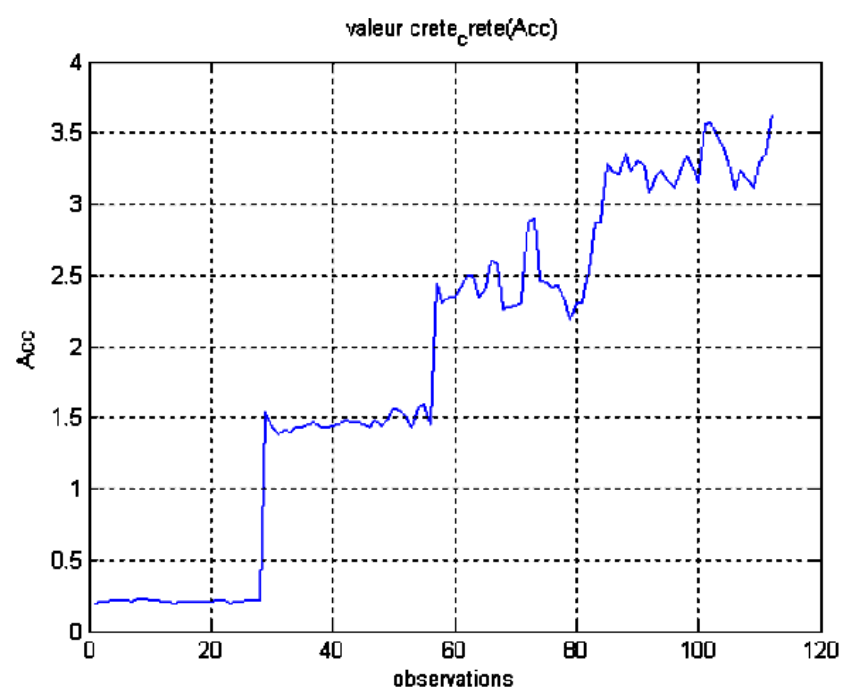

Fig. 6. Variation indicator (Acc).

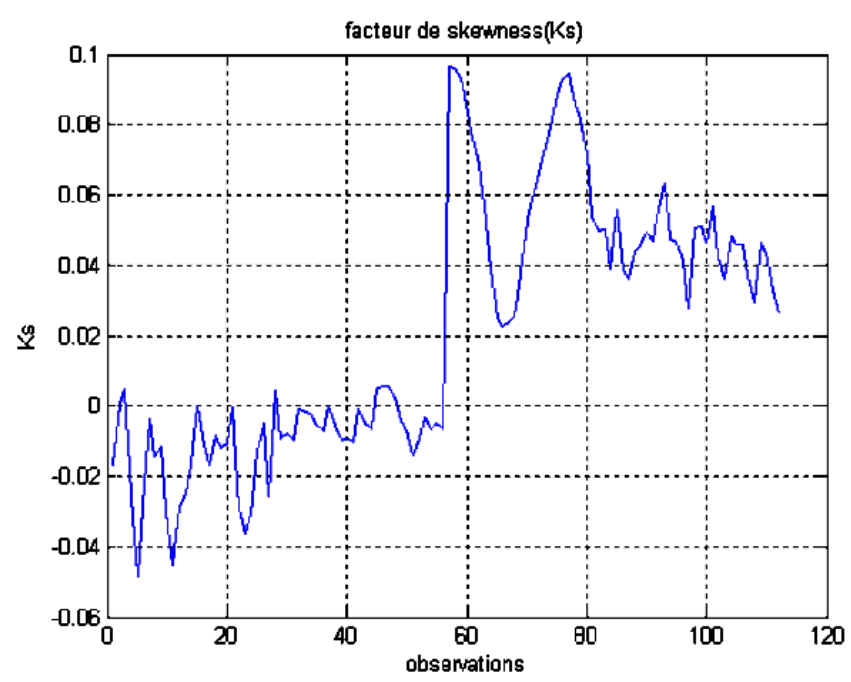

Fig. 7. Variation Indicator (Ks).

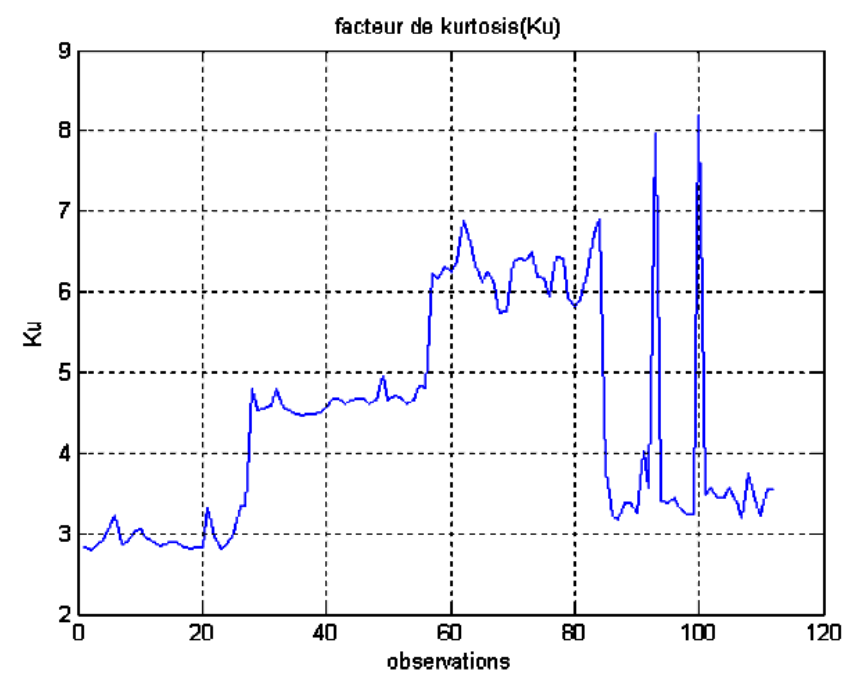

Fig. 8. Variation indicator $(\mathrm{Ku})$.

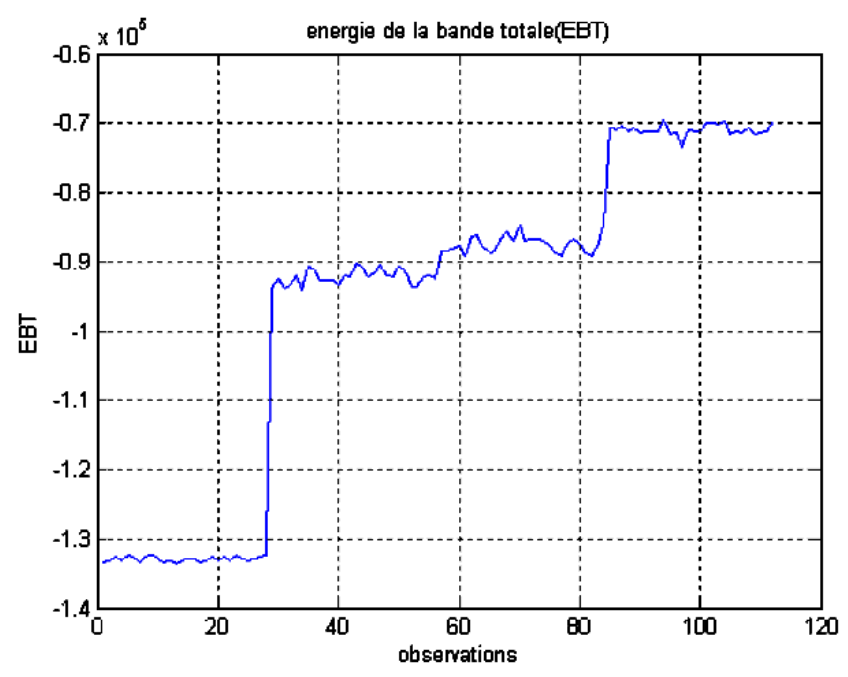

Fig. 9. Variation indicator (EBT). 


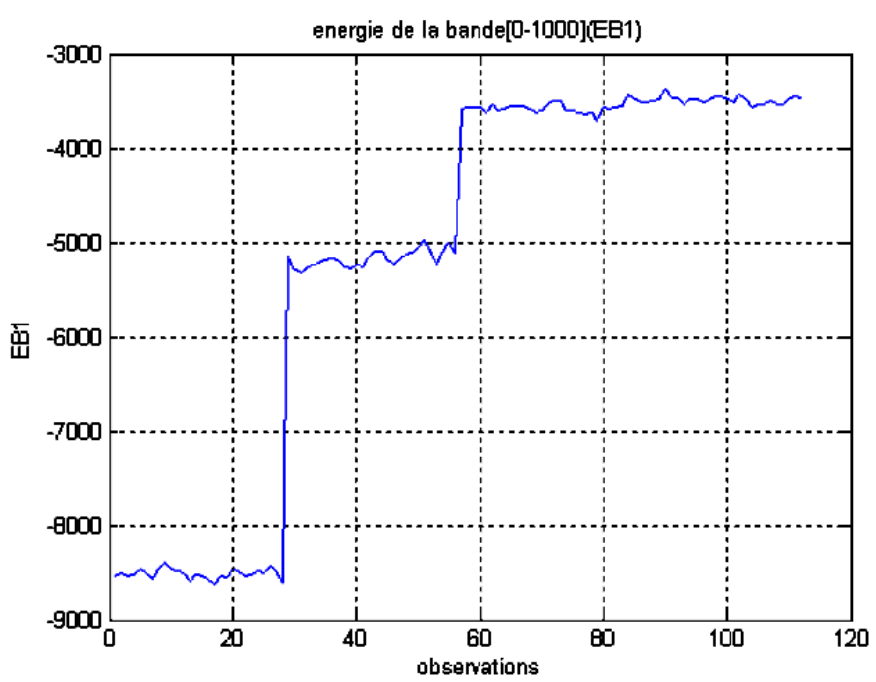

Fig. 10. Variation indicator (EB1).

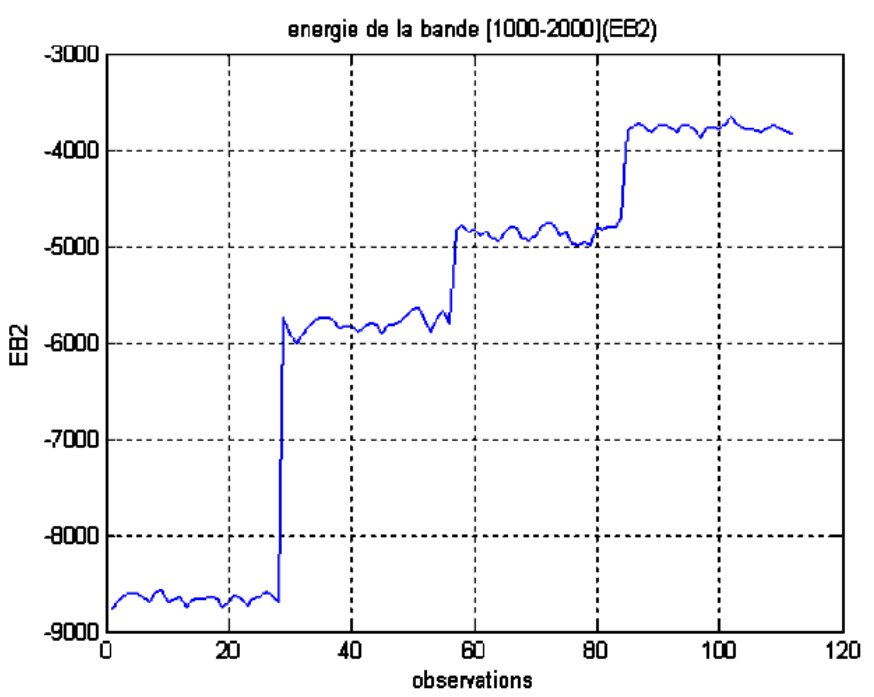

Fig. 11. Variation indicator (EB2).

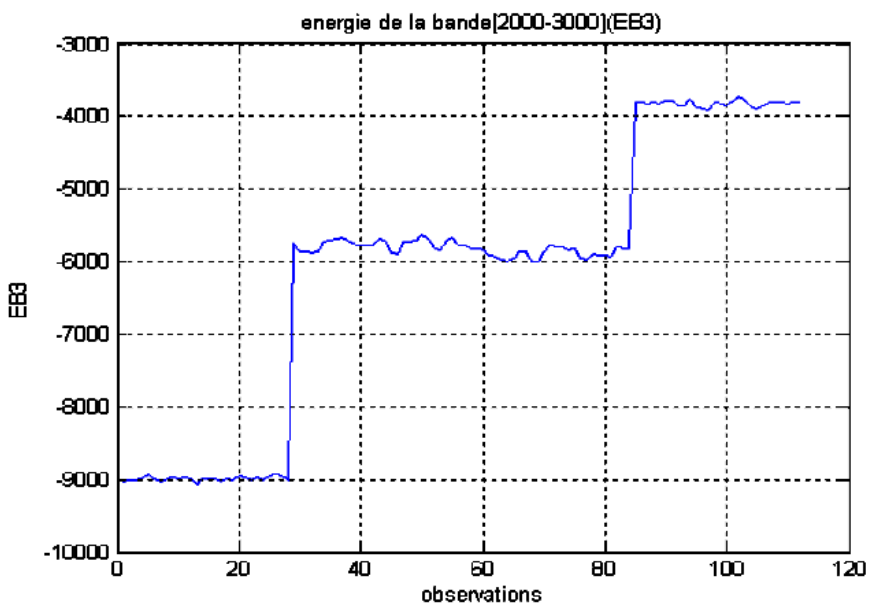

Fig. 12. Variation indicator (EB3).

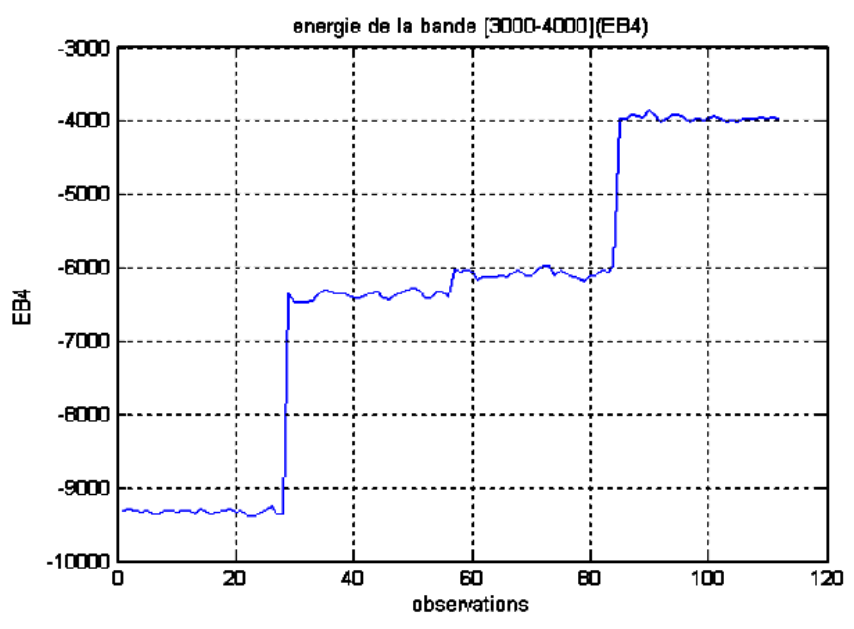

Fig. 13. Variation indicator (EB4).

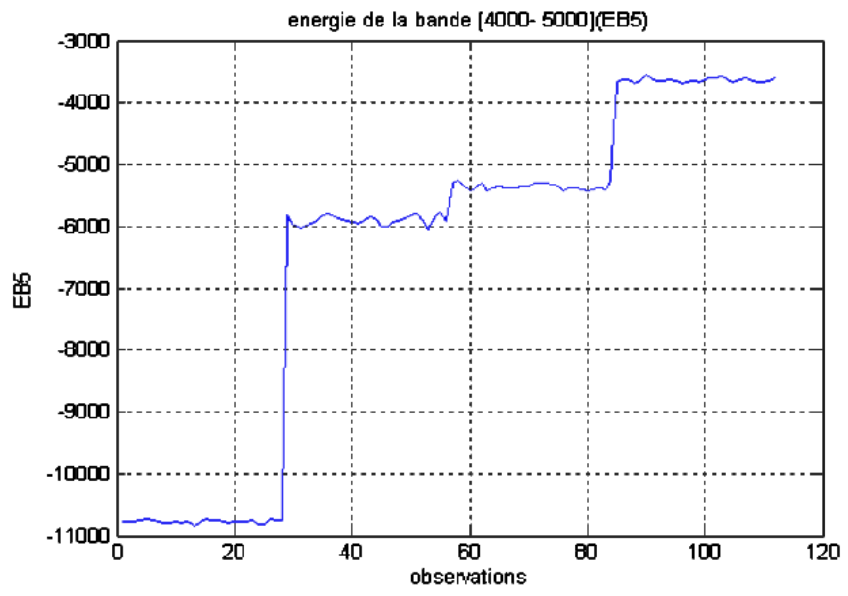

Fig. 14. Variation indicator (EB6).

for the classification of defects in bearings. The data that must classify and treat are stored in an array of type observations/variables of the form:

$$
\text { database }=\left(\begin{array}{ccc}
x_{11} & x_{12} & \ldots x_{1 M} \\
x_{21} & x_{22} & x_{2 M} \\
\vdots & \ddots & \vdots \\
x_{N 1} & x_{N 2} \ldots & x_{N M}
\end{array}\right)=\left[\begin{array}{c}
X_{1} \\
X_{2} \\
\vdots \\
X_{N}
\end{array}\right]
$$

where $\left\{\begin{array}{l}N \text { is the number of observations } \\ M \text { is the number of indicators }\end{array}\right.$

We then have:

$M$ : indicators, shown in columns, and $N$ observations represented on lines will be divided into $N c$ classes $(w 1$, $\left.w 2, \ldots W_{N C}\right)$ or operating modes, each class $w_{\mathrm{i}}$ has $N_{\mathrm{i}}$ observations.

For our work:

$N=112$ observations (28 slices per 4 signals).

$M=3$; the number of indicators that represent the input variables of the fuzzy inference system, we choose three most relevant indicators calculated from the 11 indicators (this choice is justified in a previous section). 
Table 1. Fuzzy sets (classes) of the variable output (state).

\begin{tabular}{ll}
\hline Fuzzy sets (classes) & $\begin{array}{l}\text { Diameter of the fault (inch) } \\
\text { with the output variable }\end{array}$ \\
\hline$N 1$ & No fault \\
$N 2$ & 0.007 \\
$N 3$ & 0.021 \\
$N 4$ & 0.028 \\
\hline
\end{tabular}

In addition this number is sufficient because every time we increase the number of input variables and the number of membership functions associated with each variable. We will have a complexity for the generation of fuzzy inference rules, for example: for two input variables and four membership functions for each of the two variables, we have $\left(4^{2}=16\right)$ fuzzy inference rules, and for three input variables and four membership functions, we have $\left(4^{3}=64\right)$ rules. The three indicators are selected, a type of statistic: the STDEV (ET) (this indicator is widely used for fault monitoring bearings), and the other two types of frequency (EB2) and (EB5) that represent the outcome of the energy bands [1000-2000 Hz], [4000$5000 \mathrm{~Hz}$ ] (these indicators are also selected for the reason that they are very sensitive to the variation of energy (in our case we have an increase of the size of the defect and therefore an increase in energy)).

\subsection{Choice of classes (output of fuzzy sets (FIS))}

We opted for the system output (FIS) a single output variable, which represents the health of the studied device (state). For this variable we chose four fuzzy sets corresponding to different diameters of default. Table 1 shows the different fuzzy sets (classes) associated with the output variable.

\section{Conduct of classification by fuzzy inference system (FIS)}

The methodology chosen for the classification of different states of machine faults by a fuzzy inference system (FIS) for the diagnosis is represented by the flowchart in Figure 15.

\section{Implementation of fuzzy inference system (FIS) in Matlab}

The implementation of fuzzy inference system in Matlab can be achieved by two different methods, either using the control toolbox (Fuzzy Logic) or the graphical user interface of the latter [10].

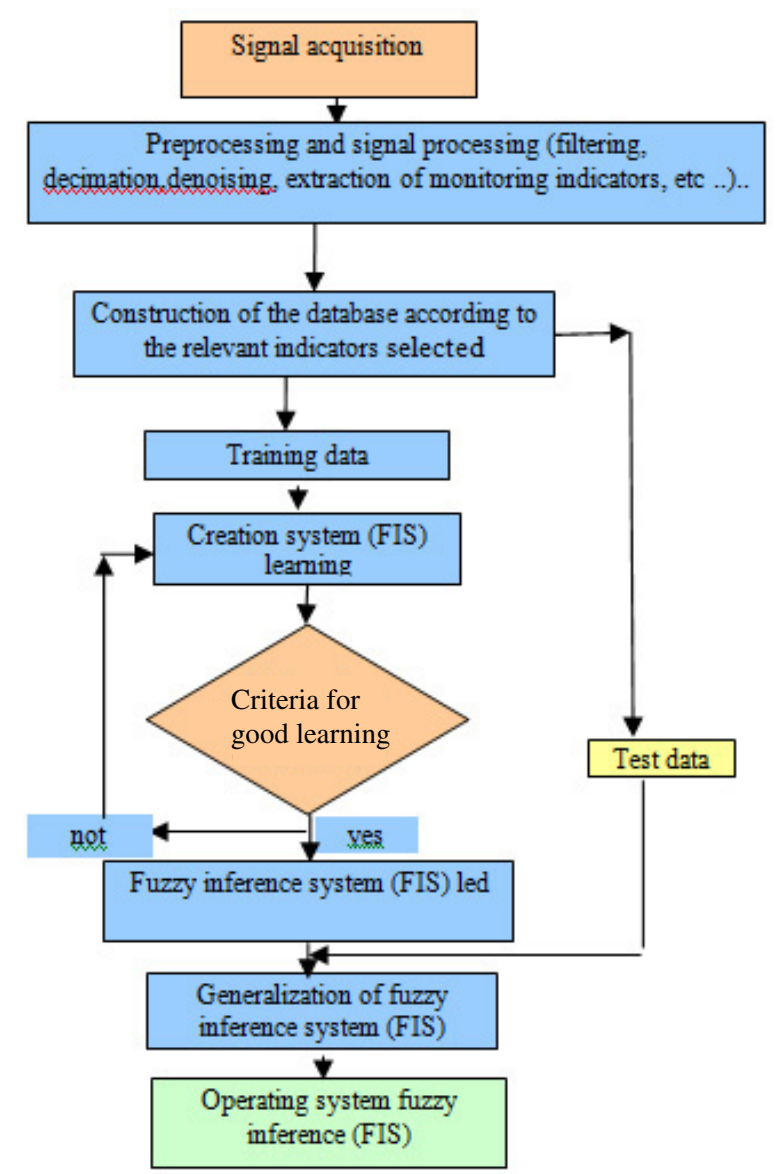

Fig. 15. Flowchart of implementation of the fuzzy inference system (FIS) for the classification of defects.

\subsection{Implementation system (FIS) through the graphic interface}

\subsubsection{Structure of the fuzzy inference system (FIS)}

We have chosen a fuzzy inference system of Mamdani type with three variables at the input and a variable to the output. Figure 16 shows the block diagram of our system. We chose a fuzzy inference mechanism that works with the methods shown in Figure 16, which should lead to a single value of the variable output (state). These methods are:

- And méthode: method used for the AND operator (min).

- Or méthode: method used for the OR operator (Max).

- Imp méthode: implication method (min).

- Agg méthode: method of aggregation rules (max).

- Deffuz méthode: method of defuzzification (centroid).

\subsubsection{Fuzzification of the input variables and output}

Figures 17-20 respectively represent the fuzzification of the three input variables (ET, EB2, EB5) and the output variable (state). 


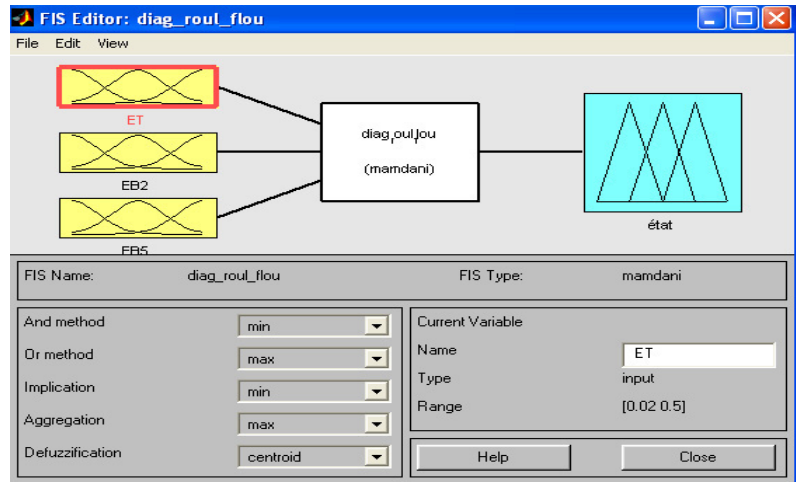

Fig. 16. Block diagram of (FIS).

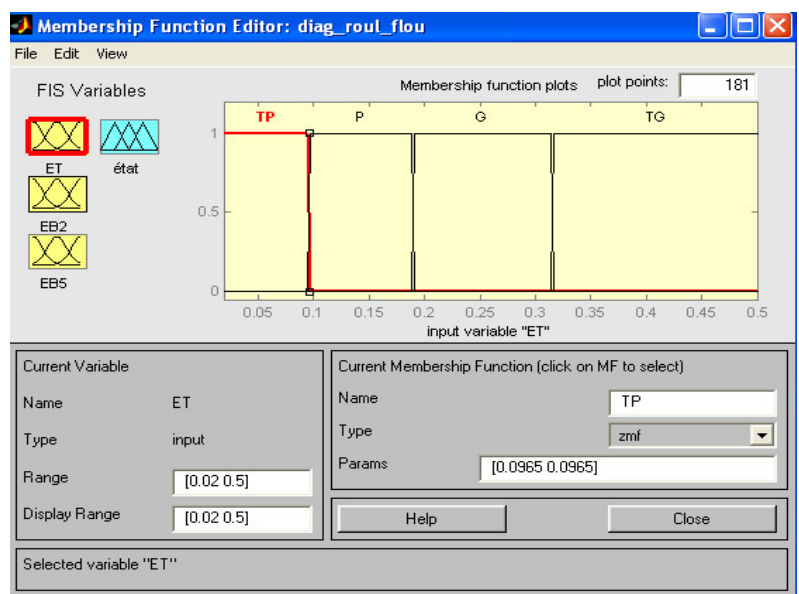

Fig. 17. Fuzzification of the input variable "the STDEV (ET)".

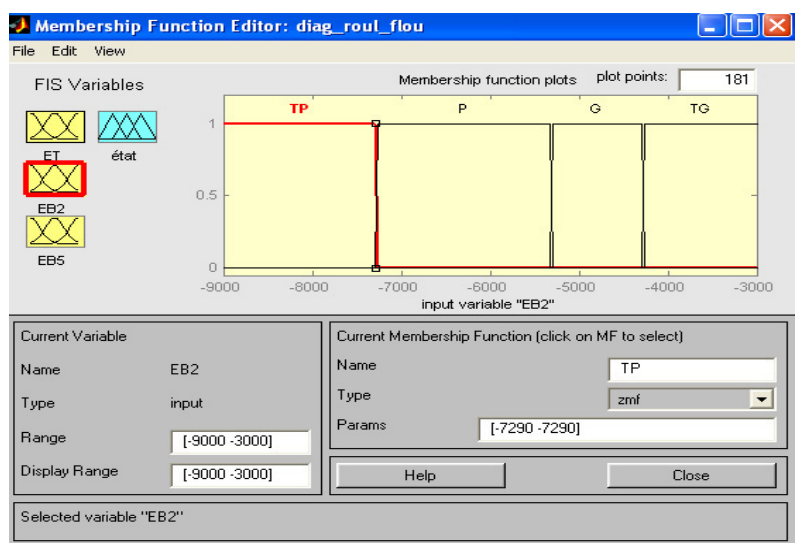

Fig. 18. Fuzzification of the input variable "energy band [1000-2000 Hz] (EB2)".

\subsubsection{Inference rules}

The different possible rules according to the fuzzification of input variables and output are selected (three input variables and 4 functions for each variable). We obtain 64 rules of inference $\left(4^{3}=64\right)$ are presented partly on the right.

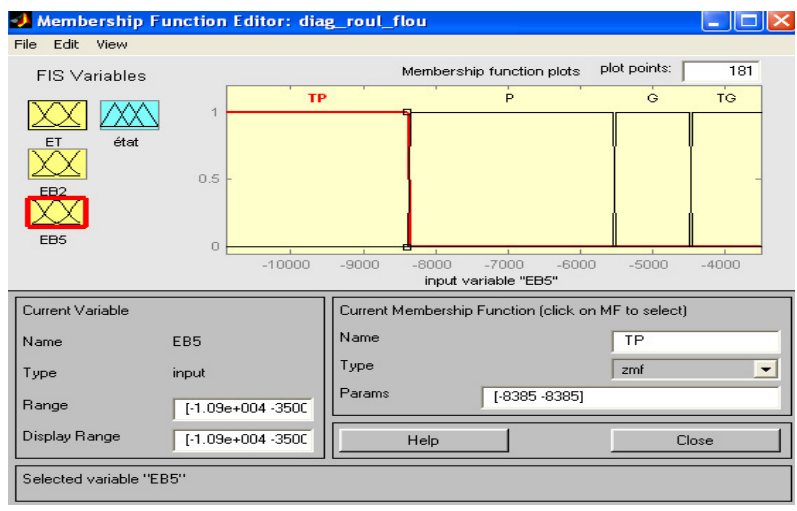

Fig. 19. Fuzzification of the input variable "energy band [4000-5000 Hz] (EB5)".

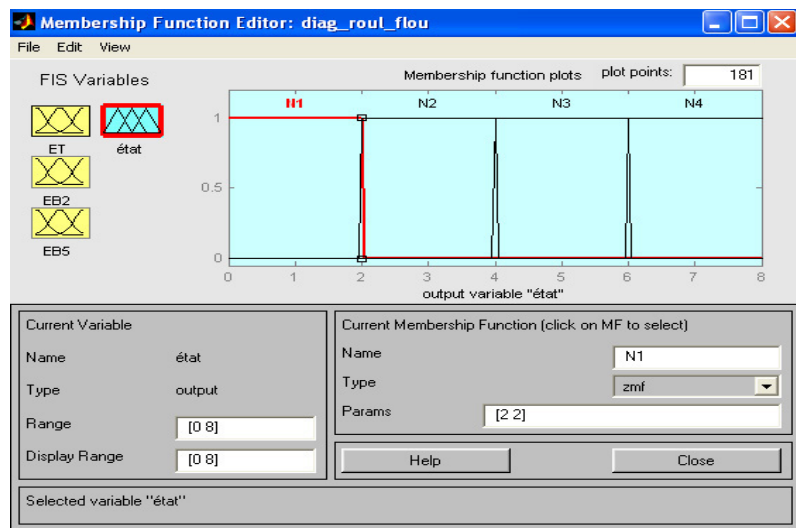

Fig. 20. Fuzzification of the output variable "Device status (state)".

1. IF [(ET is TP) and (EB2 is TP) and (EB5 is TP)] THEN (condition is N1) (1) 2. IF [(ET is TP) and (EB2 is TP) and (EB5 is P)] THEN (condition is N2) (1) 3. IF [(ET is TP) and (EB2 is TP) and (EB5 is G)] THEN (condition is N3) (1)

4. IF [(ET is TP) and (EB2 is TP) and (EB5 is TG)] THEN (condition is N4) (1) 5. IF [(ET is TP) and (EB2 is P) and (EB5 is TP)] THEN (condition is N1) (1) 6. IF [(ET is TP) and (EB2 is $\mathrm{P}$ ) and (EB5 is $\mathrm{P})]$ THEN (condition is N2) (1) 7. IF [(ET is TP) and (EB2 is P) and (EB5 is G)] THEN (condition is N3) (1) 8. IF [(ET is TP) and (EB2 is P) and (EB5 is TG)] THEN (condition is N4) (1) 9. IF [(ET is TP) and (EB2 is G) and (EB5 is TP)] THEN (condition is N3) (1) 10. IF [(ET is TP) and (EB2 is G) and (EB5 is P)] THEN (condition is N3) (1)

55. IF [(ET is TG) and (EB2 is $\mathrm{P})$ and (EB5 is G)] THEN (condition is N4) (1) 56. IF [(ET is TG) and (EB2 is P) and (EB5 is TG)] THEN (condition is N4) (1) 57. IF [(ET is TG) and (EB2 is G) and (EB5 is TP)] THEN (condition is N4) (1) 58. IF [(ET is TG) and (EB2 is G) and (EB5 is P)] THEN (condition is N4) (1) 59. IF [(ET is TG) and (EB2 is G) and (EB5 is G)] THEN (condition is N4) (1) 60. IF [(ET is TG) and (EB2 is G) and (EB5 is TG)] THEN (condition is N4) (1)

61. IF [(ET is TG) and (EB2 is TG) and (EB5 is TP)] THEN (condition is N4) (1) 62. IF [(ET is TG) and (EB2 is TG) and (EB5 is P)] THEN (condition is N4) (1) 63. IF [(ET is TG) and (EB2 is TG) and (EB5 is G)] THEN (condition is N4) (1) 64. IF [(ET is TG) and (EB2 is TG) and (EB5 is TG)] THEN (condition is N4) (1) 


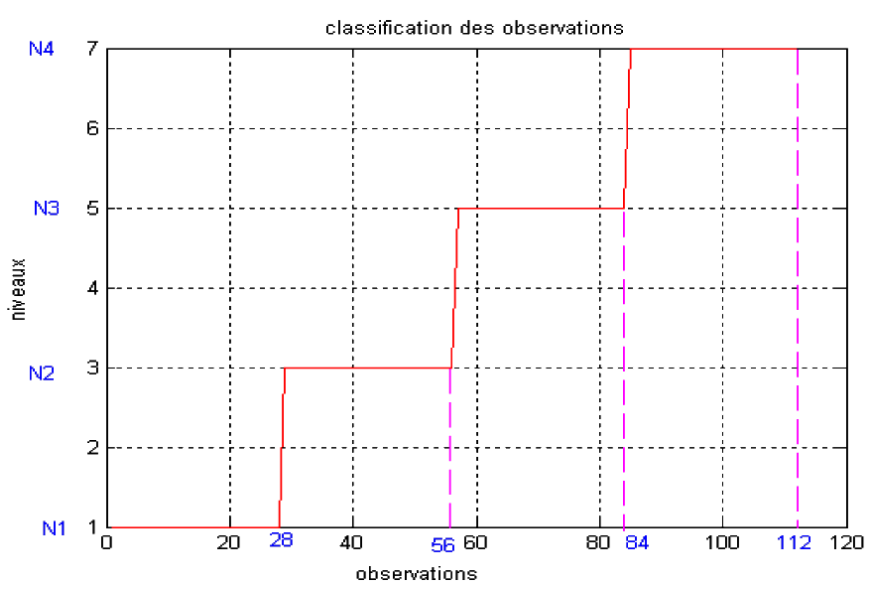

Fig. 21. Classification of the database (bearings).

\subsection{Classification of the database by the fuzzy inference system (FIS)}

We have designed a program in the Matlab environment using the controls in the toolbox "fuzzy logic" for the implementation of fuzzy inference system (FIS), developed. The program also allows to classify the observations database. The Appendix presents the results of the classification database.

\section{Results and discussion}

\subsection{Graphical representation of results classification}

Figure 21, graphically represents the result of classification, we note that:

- All the observations considered at the start of class 1 (no defects) that correspond to the line 1 on line 28 in the data matrix, are classified by the system at $N 1$, that is to say in the class 1 .

- All the observations considered at the start of class 2 (diameter of the defect (0.007 inch)) that correspond to the line 29 on line 56 in the data matrix, are classified by the system at $N 2$, that is to say in the class 2 .

- All the observations considered starting from the class 3 (diameter of the defect $(0.021$ inch)) that correspond to the line 57 on line 84 in the data matrix, are classified by the system at $N 3$, it is say in class 3 .

- All the observations considered starting from the classe 4 (diameter of the defect $(0.028$ inch $)$ ) that correspond to the line 85 on line 112 in the data matrix, are classified by the system at the $N 4$, that is, say in class 4 .

\subsection{Interpretation of results classification}

The result obtained by the classification system (FIS) is coincident with the hypothesis that we considered in Section 3.3, and the distribution of the database into four classes, class 1: no default, class 2: with defect (0.007 inch of $\Phi)$, class 3 : with default ( 0.021 inch of $\Phi)$, and class 4 : with default $(0.028$ inch of $\Phi)$. Note that all observations of the database are classified correctly, which gives a correct classification rate of $100 \%$. The high rate of good classification system may be due to:

- The right choice of system parameters (input variables and output, number and type of membership functions chosen for the different variables, rules of inference, defuzzification method), especially the three input variables system (ET, EB2, EB5), these variables (indicators) are most relevant for classification among 11 indicators initially calculated.

- The preprocessing applied to the various slices of the signals studied, and the calculation of indicators in frequency bands neither too wide nor too narrow, provide the indicators sensitive to changes that may occur on the system studied.

- The total separation of the four classes may also be due to the size of the defects created on the system, which are relatively spaced apart (the spacing of the dimensions of the defects produced energy nival separated) In the end, we can say that the fuzzy inference system (FIS), with this configuration, designed to clearly separate the four classes.

\section{Conclusion}

The difficulty is always in this type of diagnosis by fuzzy inference systems in the choice of parameters, starting with the choice of input variables (vector form), through the number of fuzzy sets associated with each variable input and output arriving in the edition of the rules especially if the number of fuzzy sets associated with different input variables is large enough. It can be said here that there are no rules for choosing the parameters of the inference system, which vary depending on the problem studied. Only the user experience can overcome this difficulty. The implementation of fuzzy inference system (FIS) in the Matlab environment is characterized by its simplicity, a just choice of the parameters of the system, and then an implement either using GUI or from command in Matlab box.

\section{Appendix: Classification result of the database of bearings by the classifier based on fuzzy inference}

\section{- Note:}

This appendix contains the actual values of the output variable that represents the state of the device studied. So we have:

Line: one line, which represents the real value of output variable. 
Column: 1 to 112 of the columns are labeled observations of the database.

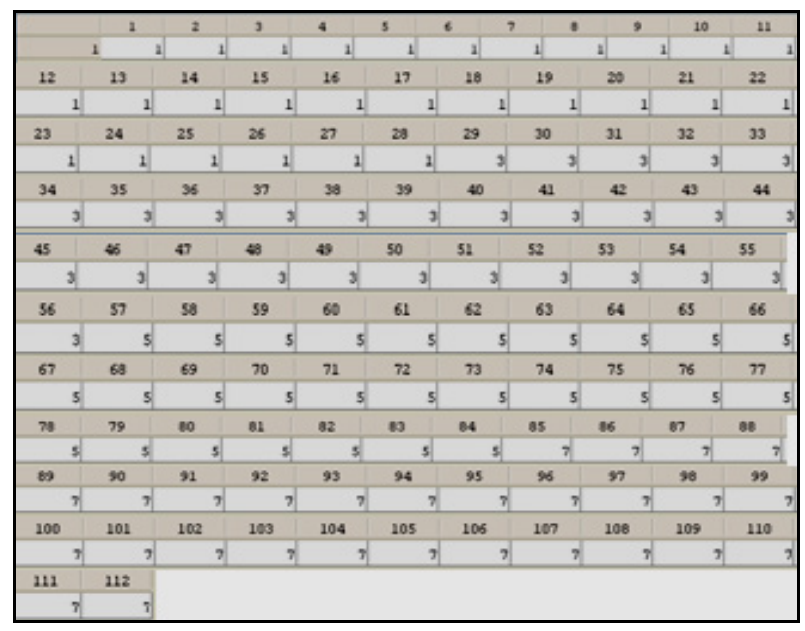

\section{References}

[1] T. Liu, J.H. Singonahalli, N.R. Iyer, Detection of roller bearing defects using expert systems and fuzzy logic, Mech. Syst. Signal Process. 10 (1996) 595-614

[2] C.K. Mechefske, Objective Machinery Fault Diagnosis Using Fuzzy Logic, Mech. Syst. Signal Process. 12 (1998) $885-862$

[3] X. Lou, K.A. Loparo, Bearing fault diagnosis based on wavelet transform and fuzzy inference, Mech. Syst. Signal Process. 18 (2004) 1077-1095
[4] V. Sugumaran, K.I. Ramachandran, Automatic rule learing using decision tree for fuzzy classifier in fault diagnosis of roller bearing, Mech. Syst. Signal Process. 21 (2007) $2237-2247$

[5] T. Boutros, M. Liang, Mechanical fault detection using fuzzy index fusion, Int. J. Machine Tools Manuf. 47 (2007) 1702-1714

[6] J.-D. Wu, C.-C. Hsu, Fault gear identification using vibration signal with discrete wavelet transform technique and fuzzy-logic inference, Expert Syst. Appl. 36 (2009) $3785-3794$

[7] N. Saravanan, S. Cholairajan, K.I. Ramachandran, Vibration-based fault diagnosis of spur bevel gear box using fuzzy technique, Expert Syst. Appl. 36 (2009) 31193135

[8] J.D. Wu, C.C. Hsu, Fault gear identification using vibration signal with discrete wavelet transform technique and fuzzy logic inference, Expert Syst. Appl. 36 (2009) $3785-3794$

[9] C. Aliustaoglu, H. Metin Ertunc, H. Ocak, Tool wear condition monitoring using a sensor fusion model based on fuzzy inference system, Mech. Syst. Signal Process. 23 (2009) 539-546

[10] K.A. Loparo, Bearings vibration data set, Case Western Reserve University, http://www.eecs.cwru. edu/laboratory/bearing/download.htmS.

[11] Mathworks, Fuzzy Logic Toolbox-for Use with MATLABs, User manual of Mathworks, 2000 